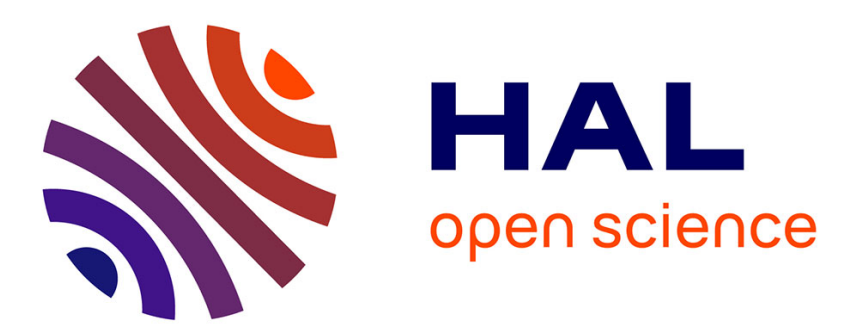

\title{
Des regards croisés sur les enjeux de la biodiversité dans la gestion forestière
}

Christian Barthod, Jacques Andrieu

\section{To cite this version:}

Christian Barthod, Jacques Andrieu. Des regards croisés sur les enjeux de la biodiversité dans la gestion forestière. Revue forestière française, 2011, 63 (5), pp.503-508. 10.4267/2042/46114 . hal03449729

\section{HAL Id: hal-03449729 \\ https://hal.science/hal-03449729}

Submitted on 25 Nov 2021

HAL is a multi-disciplinary open access archive for the deposit and dissemination of scientific research documents, whether they are published or not. The documents may come from teaching and research institutions in France or abroad, or from public or private research centers.
L'archive ouverte pluridisciplinaire HAL, est destinée au dépôt et à la diffusion de documents scientifiques de niveau recherche, publiés ou non, émanant des établissements d'enseignement et de recherche français ou étrangers, des laboratoires publics ou privés. 


\title{
DES REgARDS CROISÉS \\ SUR LES ENJEUX DE LA BIODIVERSITÉ DANS LA GESTION FORESTIÈRE
}

\author{
Christian Barthod - Jacques Andrieu
}

L’Assemblée générale des Nations unies a fait de 2010 l'année internationale de la biodiversité, et de 2011 l'année internationale des forêts. Est-ce le hasard qui a présidé à un tel rapprochement, ou bien est-ce aussi la reconnaissance que la forêt est, au niveau mondial comme au niveau de la plupart des massifs, le lieu d'une grande biodiversité et généralement un espace où les mécanismes fonctionnels découlant de cette biodiversité sont encore relativement préservés ? Quoi qu'il en soit, la Revue forestière française a décidé de célébrer la conjonction de ces deux années internationales dans le présent numéro spécial.

Dans les articles qui ont été sollicités, à l'exception du patrimoine ultramarin souvent méconnu, il n'a pas été envisagé de présenter les caractéristiques de la biodiversité dans les forêts françaises, autrement que comme un rappel succinct nécessaire aux propos des auteurs. On ne trouvera donc pas de chiffres détaillés, de description précise des fonctionnalités écologiques, de bilan des recherches scientifiques en cours ni de liste d'espèces ou de milieux remarquables ou menacés. Presque vingt ans après l'adoption, à Rio de Janeiro, en 1992, de la Convention mondiale sur la diversité biologique en même temps que deux textes sur la forêt ${ }^{(1)}$, ce numéro spécial est l'occasion de faire un premier bilan de ce qui a changé, tout d'abord dans le regard sur la biodiversité en forêt, regard porté par les institutions, les gestionnaires, et plus généralement la société française, et ensuite dans les diverses initiatives en cours ou à venir concernant la forêt.

Sur des thématiques aussi complexes que la biodiversité, la connaissance représente un enjeu important, et les forestiers se sentent parfois démunis pour identifier les " sachants » qui prendront en charge leur problème, ou mieux les experts à qui s'adresser pour engager un dialogue prenant en compte les deux savoirs interdépendants de la foresterie et de la biodiversité. Il existe encore un vaste champ à explorer dans le domaine des inventaires de biodiversité, comme dans le domaine de la compréhension du fonctionnement et des dysfonctionnements des écosystèmes forestiers et associés. Mais faire appel aux connaissances d'autrui ou se risquer à innover supposent généralement que le décideur identifie un problème pour lequel la palette de ses modes opératoires traditionnels le laisse démuni, ou le conduit à identifier une forte incertitude qu'il ne peut ou ne veut pas assumer seul. En effet pour voir, il faut d'abord entrevoir ; en matière de biodiversité, plus encore qu'ailleurs, c'est indispensable.

Par ailleurs la biodiversité n'est pas une thématique simple, où il suffirait d'appliquer une grille de raisonnement prédéfinie et deux ou trois recettes pour avoir un résultat satisfaisant, nonobstant le

(1) La « Déclaration non juridiquement contraignante mais faisant autorité pour un consensus mondial sur la gestion, la conservation et l'exploitation durable de tous les types de forêts » et le chapitre forestier de l'Agenda 21. 
fait que les critères d'évaluation a priori et a posteriori sont loin d'être toujours simples ${ }^{(2)}$. Elle est certes un " objet scientifique ", requérant connaissance, rigueur et analyse, mais elle n'est pas que cela : elle est aussi un " objet culturel ", partie intégrante d'une grille de valeurs, d'une "vision du monde ». Certains voient dans les débats sur la biodiversité un luxe de pays riches, dont la prise en compte pourrait se révéler un facteur handicapant pour la maximisation de la production de biens. D’autres présentent la biodiversité, dans son état actuel (connu, mais aussi méconnu et inconnu), comme la base intangible de toute production de biens et de services, la condition indispensable et " invisible » des processus de production, dont on ne perçoit l'importance qu'en situation de déséquilibre ou de perturbation grave.

C'est pourquoi un parti a été adopté dans ce numéro : demander à deux ou trois personnalités d'horizons et d'expériences diverses, ouvertes au dialogue, de collaborer sur une question, de confronter leurs savoirs et leurs analyses, ce qui les réunit comme ce qui les différencie, afin de tenter une réponse à la fois la plus rigoureuse possible, mais aussi nuancée et ouverte sur l'avenir et sur ce qui nous échappe.

Le numéro spécial s'ouvre sur les initiatives prises sur le terrain ${ }^{(3)}$, par tous ceux qui tentent de tirer des conséquences pratiques, sur un espace donné et dans une temporalité liée à leurs objectifs et à leurs contraintes, des orientations techniques et valeurs promues par les textes nationaux et internationaux sur la gestion durable et sur la biodiversité. Il convient en effet de leur rendre un hommage tout particulier, car ils engagent du temps, de l'énergie, des convictions, et parfois de l'argent, dans un environnement professionnel et social qui ne leur est pas toujours acquis d'avance. Les vrais progrès dans les esprits et dans les faits procèdent toujours de cette confrontation laborieuse entre les théories et la pratique, entre le discours et l'opérationnel, jusqu'à trouver une certaine harmonie entre les deux et la force d'une quasi-évidence. Roland Burrus et Jean-Michel Mourey (in "Initiatives et créativité des forestiers en matière de biodiversité ») illustrent la richesse et la diversité des initiatives, individuelles et collectives, prises dans le cadre des orientations officielles et des messages de développement qui arrivent aux gestionnaires, comme d'ailleurs parfois en marge de ces orientations et de ces messages, dans les espaces de liberté que chaque gestionnaire connaît et protège jalousement.

L'irruption assez brutale de la thématique de la biodiversité dans le paysage culturel forestier s'est faite entre 1992 et 1997 , dans le sillage certes de la CNUED (4), mais surtout de la directive communautaire Habitats, faune, flore, du vif conflit opposant le ministère chargé de sa mise en œuvre et le "groupe des neuf». Ce n'est pas le lieu de pointer les raisons qui ont conduit à cet affrontement dont la responsabilité est sans doute partagée, par une escalade d'incompréhensions successives. Mais il est impossible de faire l'impasse sur la manière dont se sont mêlées inextricablement et conflictuellement les considérations techniques, juridiques, économiques, politiques et culturelles dans le premier grand débat public national sur la biodiversité impliquant la forêt après 1992. Il est d'autant plus intéressant de regarder comment s'est construite progressivement une approche plus intégratrice dans la gestion forestière au sein du réseau Natura 2000. François Bland et Lucile Rambaud (in « Le réseau Natura 2000 : un défi pour la gestion multifonctionnelle des forêts ") montrent la manière dont, à partir d'une situation très tendue, se sont progressivement élaborés de nouveaux compromis et équilibres au cours des dix dernières années, sans éluder les questions qui restent pendantes et sensibles.

Si les sites Natura 2000 sont bien un élément phare du patrimoine naturel métropolitain, en même temps que le lieu d'une responsabilité particulière de la France vis-à-vis des politiques

(2) Cf. les débats sur l'état de conservation favorable des espèces et des habitats au sein de la directive Habitats, faune, flore de 1992, ou même simplement les conditions d'une population viable.

(3) Dans une activité directe de gestion ou dans le travail de développement auprès des gestionnaires eux-mêmes.

(4) Conférence des Nations unies sur l'environnement et le développement (Rio de Janeiro, 1992). 
communautaires, la biodiversité exceptionnelle et l'ampleur de la responsabilité nationale vis-àvis des enjeux mondiaux de conservation de la biodiversité sont deux caractéristiques éminentes des forêts des douze territoires de l'outre-mer français. Dans ces forêts se cherche encore un équilibre entre d'une part la satisfaction des usages locaux, traditionnels ou liés au développement économique, et d'autre part des essais d'adaptation des outils de protection et de gestion issus de la métropole, dépendant de l'histoire, du statut juridique du territoire, mais aussi des modes de gouvernance locaux, notamment, dans certains cas, ceux des populations tirant traditionnellement leur subsistance de la forêt. Jacques Trouvilliez et Frédéric Mortier (in " Les forêts en outre-mer : un enjeu mondial pour la biodiversité ») décrivent la grande variabilité des forêts ultramarines, leur grande richesse en même temps que leur fragilité devant des menaces fortes, réelles et diverses. Conserver cette biodiversité est un devoir, en même temps qu'une évidence pour les forestiers et les naturalistes qui découvrent, gèrent ou protègent en l'état ces forêts. Mais un tel choix conduit nécessairement à devoir se poser des questions techniquement, socialement et éthiquement complexes, à une échelle et avec une acuité que l'on peine déjà à assumer en métropole.

De par sa sensibilité au long terme, le secteur forestier a été très rapidement réceptif aux interrogations sur les conséquences du changement climatique. Mais les réflexions en cours sont liées aux valeurs accordées à la biodiversité, aux arbres par rapport à l'ensemble des autres espèces vivantes, aux fonctions écologiques. Elles bousculent nécessairement les visions fixistes de la biodiversité, selon des modalités que les forestiers perçoivent bien, sans pouvoir en tirer toutes les conséquences. Elles obligent à prendre en compte aussi bien les évolutions tendancielles que les événements brutaux dans la composition des communautés végétales. Elles conduisent à se reposer de manière neuve de vieilles questions, telles que la variabilité génétique intra-peuplement, ou les transferts d'essences ou de provenances. C'est pourquoi les débats forestiers sur le changement climatique rencontrent souvent, de façon visible ou en creux, ceux sur la biodiversité, dans un contexte où les travaux scientifiques ne sont pas pléthore. François Lefèvre, Hervé Le Bouler et Bernard Roman-Amat (in " Changement climatique attendu et biodiversité en forêt ") se risquent à poser les termes possibles de l'interdépendance entre biodiversité, changements climatiques et forêts.

Très rapidement dans beaucoup de discussions forestières, la préservation de la biodiversité est appréciée comme quelque chose qui, consubstantiellement, coûte. Le débat s'oriente alors vers la recherche d'une optimisation entre les fonctions économiques, écologiques et sociales de la forêt, en fait de la gestion forestière, en pondérant les demandes sociales perçues comme faiblement solvables, les besoins prévisibles des marchés connus et les contraintes de toutes natures des décideurs. S'esquissent comme contrepoids possible à cette perception toutes les recherches visant à donner une valeur économique à la biodiversité, notamment celles de certains économistes des ressources naturelles qui visent à positionner la biodiversité, comme condition et facteur de production. Ces débats sont encore balbutiants, mais riches et passionnés. Mais souvent les décideurs forestiers, peu initiés aux travaux scientifiques sur l'économie des ressources naturelles, confondent le prix et la valeur, sous-estiment la pluralité des types d'optimisation possibles, ignorent la dimension culturelle et philosophique d'une actualisation économique, et peinent à comparer des "systèmes forestiers " inégaux sur le plan de la biodiversité. Jean-Philippe Terreaux et Jacques Weber (in "Réflexions sur l'évaluation de la biodiversité et l'aménagement des forêts ") présentent une initiation à ces approches novatrices, où la biodiversité et économie semblent pouvoir parler un langage commun.

Au niveau des penseurs internationaux de la biodiversité s'est imposée en peu de temps l'idée que les services écosystémiques procurés par la forêt à nos sociétés pouvaient offrir une piste pédagogique pour valoriser les fonctions non marchandes de la forêt auprès des grands déci- 
deurs politiques et budgétaires. Il existe de nombreuses initiatives internationales, communautaires et nationales, étatiques ou non étatiques, cherchant à promouvoir parallèlement ce concept de services écosystémiques offerts par la forêt, à leur donner une valeur économique et enfin à encourager le développement d'outils de marché transformant cette valeur économique en valeur monétaire pour des décideurs locaux. Le succès rencontré par l'idée de paiement pour services environnementaux (PES), auprès des propriétaires et gestionnaires forestiers, est extraordinaire un peu partout dans le monde, et les initiatives foisonnent. Mais cet engouement fait souvent oublier les difficultés sémantiques du débat, et le fait qu'il faut à la fois un problème ressenti et une motivation à agir pour surmonter toutes les difficultés d'une telle démarche. Passer de la valeur économique à la valeur marchande ne va pas de soi, et les expériences réussies montrent que le consentement à payer est souvent plus important que l'évaluation économique préalable. À partir d'une riche expérience internationale scientifique et pratique, Bernard Chevassus-au-Louis et Romain Pirard (in "Les services écosystémiques des forêts et leur rémunération éventuelle ») recadrent avec rigueur les termes nécessaires d'une approche dont le succès médiatique a fait oublier les limites.

Les naturalistes et les associations de protection de la nature sont souvent mal à l'aise devant ces essais de valorisation économique de la biodiversité, et rappellent avec d'autant plus de force les fondements éthiques qui devraient présider aux politiques de préservation de la biodiversité. Le contrepoids à une approche matérialiste, utilitariste et anthropocentrée engage nécessairement des valeurs et des références culturelles, explicites ou implicites, largement partagées ou élitistes, tout comme, d'ailleurs, l'approche contre laquelle elle se dresse. L'approche des paysages forestiers, à l'articulation entre l'écologie, la culture et l'esthétique, partagée entre la quête revendiquée d'objectivité et une subjectivité inévitable, permet de soulever beaucoup de présupposés philosophiques qui se cachent souvent derrière des argumentations scientifiques. En partant d'un conflit sur une forêt dans le parc national de la Vanoise pour s'interroger sur la pluralité des "écologies » auxquelles les partenaires se réfèrent, avant d'aborder "l'esthétisation de la nature », Catherine Larrère et Raphaël Larrère (in " Des paysages forestiers ») nous introduisent dans un vaste paysage sociologique et philosophique.

L'Union européenne est le lieu de réflexions intenses et d'initiatives politiques ambitieuses en matière de biodiversité, comme l'ont démontré les directives Oiseaux (1979) et Habitats, faune, flore (1992), mais aussi son positionnement dans la conférence des parties de la convention sur la diversité biologique. La Commission, le Conseil et le Parlement européen mettent conjointement en avant une volonté d'exemplarité et une forte ambition communautaire dans le domaine de la biodiversité, certes très rarement ciblées sur la forêt, mais qui sont sources d'importants effets dérivés sur les politiques forestières. À la stratégie communautaire pour la biodiversité (adoptée le 21 juin 2011), fait écho une nouvelle stratégie nationale pour la biodiversité (adoptée le 19 mai 2011), deux stratégies pour lesquelles le secteur forestier est, de manière non explicite mais nécessaire, à la fois un espace et une thématique d'application, mais aussi le lieu de partenariats à créer ou renforcer. Robert Flies et Paul Delduc (in « Forêt et biodiversité : dynamiques communautaire et nationale $"$ ) font le point de ce qui se dessine dans ce domaine, tant au niveau communautaire qu'au niveau national. Au-delà du réseau Natura 2000, il est intéressant de noter la convergence des nouvelles priorités nationale et communautaire sur la connectivité écologique (trames verte et bleue, en France ; infrastructures vertes, en vocabulaire communautaire), et sur les territoires ultramarins.

Le débat sur la préservation de la biodiversité est mondial, menée essentiellement mais pas exclusivement dans le cadre de la convention sur la diversité biologique, avec une revendication plus ou moins assumée d'aborder l'ensemble des politiques sectorielles qui ont des incidences, positives ou négatives, sur la biodiversité. Les débats internationaux sur le changement climatique 
ont conduit à la création de nouveaux instruments forestiers, comme REDD+, qui cristallise les espoirs comme les difficultés relatives à la place de la biodiversité dans les politiques liées au changement climatique. Les espoirs nés à Rio de Janeiro, en 1992, d'un instrument juridique non contraignant sur les forêts au plan mondial sont aujourd'hui largement déçus. Le processus des conférences ministérielles pour la protection des forêts en Europe (Forest Europe, né à Strasbourg en 1990), qui avait réussi à formaliser les ambitions relatives à la biodiversité et à la gestion durable des forêts pour le continent européen, semble s'engager dans la direction d'un tel instrument international pour les seuls pays européens. Le débat international forestier n'est certes plus aussi politiquement flamboyant que dans les années 1990, et prend acte d'avancées, mais aussi de déceptions. Ce constat ne doit néanmoins pas dissimuler tout ce qui se passe de concret et de passionnant dans les grands et petits projets multilatéraux ou dans la coopération bilatérale. Guillaume Choumert, Gilles Kleitz et Cyril Loisel (in « La place de la biodiversité dans la diplomatie et la coopération forestière internationale ") présentent l'actualité des débats, ainsi que les avancées et difficultés rencontrées dans les processus intergouvernementaux.

Avec le débat sur la chouette tachetée, les États-Unis d’Amérique avaient témoigné dans les années 1990 de la vigueur, voire de la violence des débats politiques sur la biodiversité, et des conséquences pratiques sur les équilibres économiques d'une région comme le Nord-Ouest des USA ou sur les orientations de la gestion des forêts publiques. Sally Collins et Hutch Brown (in "L'US Forest Service et la biodiversité ») décrivent le contexte technique et culturel dans lequel l'US Forest Service agit et se transforme depuis 15 ans, les importantes mutations internes, les paris pris et à venir. Parmi les acquis de ces dernières années, les auteurs insistent d'une part sur l'émergence du " paysage » comme lieu opérationnel de gestion de la biodiversité, notamment dans la perspective du changement climatique, d'autre part sur la manière dont une politique des services écologiques pourrait remettre les " gens " au centre des débats forestiers, valoriser le changement des pratiques et déboucher sur une rémunération contractuelle. Les forestiers, comme les environnementalistes, savent que les débats peuvent mettre 5 à 10 ans pour franchir l'Atlantique et se reformuler en fonction du contexte européen, mais réfléchir à l'avance sur l'expérience nord-américaine, pour réagir ou pour s'adapter, ne peut être que stimulant.

Le Grenelle de l'environnement a officialisé et accéléré un dialogue direct entre les organismes forestiers et les associations de protection de la nature. Personne n'ignore plus l'existence et le contenu du protocole d'accord de 2007 Produire plus de bois tout en préservant mieux la biodiversité : une démarche territoriale concertée dans le respect de la gestion multifonctionnelle des forêts. La biodiversité y figure en bonne place, mais toutes les exégèses sont encore possibles sur la manière dont, au quotidien, ce titre est concrètement cité, reformulé ou tronqué, dont certains éléments sont parfois davantage mis en valeur que d'autres. Quoi qu'il en soit, pour la première fois, un "slogan politique » et une ambition commune sont partagés par les forestiers et par les environnementalistes, et la biodiversité en est une composante. François Lefèvre, Daniel Vallauri et Emmanuelle Neyroumande (in "Biodiversité et gestion des forêts : la France à la croisée des chemins ») reviennent sur ces dix dernières années. Avec le sentiment de certains rendez-vous manqués et la crainte que ce qui semblait acquis puisse être remis en cause par de nouvelles priorités, les auteurs mettent l'accent sur ce qui semble avoir évolué dans le sens espéré par les associations de protection de la nature, mais aussi sur leurs insatisfactions, doutes et inquiétudes, bref sur tout ce qui reste au cœur des discussions en cours ou émergentes : gouvernance générale du secteur, place reconnue à la biodiversité dans les forêts de production, territoire et gouvernance, résilience des écosystèmes forestiers, naturalité des forêts...

Les responsables de la forêt privée, de la forêt communale et de la forêt domaniale revendiquent une gestion durable, qui suppose une durabilité biologique dont la biodiversité est une composante essentielle, mais aussi une durabilité économique, une durabilité institutionnelle et 
une durabilité politique. La biodiversité est à ce titre " une partie de leur paysage », mais une partie seulement. Entre les trois grands responsables nationaux qui s'expriment conjointement, il existe un socle commun qui reflète ce que l'on peut qualifier de compréhension partagée de la gestion durable à la française, mais aussi un attachement au chemin parcouru au cours des vingt dernières années en matière de prise en compte de la biodiversité dans la gestion forestière, en même temps que la conscience que les équilibres actuels ne sont pas, et ne peuvent pas être définitifs. Henri Plauche Gillon, Jean-Claude Monin et Pascal Viné (in « Biodiversité et gestion forestière ") réagissent sur le bilan dressé par FNE et le WWF et sur les attentes exprimées, mais ils mettent également en perspective leur perception des enjeux de la biodiversité dans la décennie à venir par rapport aux évolutions du contexte économique et social dans lequel les gestionnaires forestiers prennent leurs décisions. Sans cacher leurs inquiétudes sur la viabilité économique du "système forestier » actuel, et donc sur la pérennité du cadre institutionnel et politique dans lequel la biodiversité est actuellement appréhendée par les décideurs forestiers individuels, ils plaident pour le renforcement du dialogue et pour la reconnaissance des services écologiques rendus par la forêt à la société.

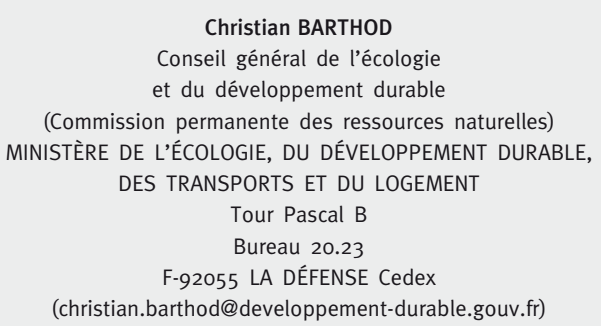

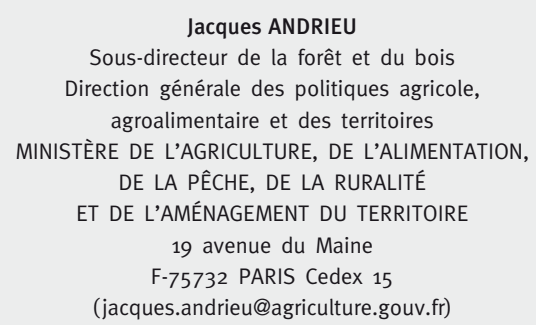

Les rédacteurs en chef invités de ce numéro spécial dégagent toute responsabilité sur les opinions émises par les co-auteurs, et souhaitent que ces éclairages variés stimulent la réflexion et la motivation des acteurs forestiers dans la quête d'une gestion durable, respectueuse de la biodiversité, créatrice de biens matériels et immatériels comme de services à la société. 